\title{
Apoptosis - Regulation and clinical implications
}

\author{
U Zangemeister-Wittke ${ }^{\star, 1}$ and H-U Simon ${ }^{\star, 2}$ \\ ${ }^{1}$ Department of Internal Medicine, Division of Medical Oncology, University Hospital, Zurich, Switzerland. ${ }^{2}$ Department of Pharmacology, University of Bern, Bern, \\ Switzerland \\ * Corresponding authors: U Zangemeister-Wittke, Division of Medical Oncology, University Hospital, Häldeliweg 4, CH-8044 Zürich. Tel: +41-1-634-2877; \\ Fax: +41-1-634-2872; E-mail: uwe.zangemeister@dim.usz.ch and H-U Simon, Department of Pharmacology, University of Bern, CH-3010 Bern, Switzerland. \\ Tel: +41-31-632-3281; Fax: +41-31-632-4992; E-mail: hus@pki.unibe.ch
}

ECDO Conference report, 8th Euroconference on Apoptosis, Davos, Switzerland, October 14-17, 2000

Apoptosis is initiated by extracellular or intracellular signals in which a complex machinery is activated to start a cascade of events ultimately leading to the degradation of nuclear DNA and dismantling of the cell. Dysregulation of apoptosis is associated with many pathologic conditions and may even be central in the pathogenesis of many diseases. Whilst too little apoptosis may result in cancer, autoimmune or other chronic inflammatory diseases, too much apoptosis is involved in stroke-induced neuronal damage and neurodegenerative disorders. Apoptosis research is rapidly proceeding making it difficult to keep track of the constant stream of newly identified proteins and molecular interactions involved in cell death regulation. The recent progress in our understanding of the regulation of apoptosis and its importance for pathogenic processes was the subject of a European Cell Death Organization (ECDO) meeting that was organized in Davos, Switzerland (October 14-17, 2000) by $\mathrm{H}-\mathrm{U}$ Simon and $\mathrm{U}$ Zangemeister-Wittke. The 8th Euroconference on Apoptosis attracted 177 participants from Europe, North America, Asia, and Australia.

\section{Regulation of apoptosis \\ Death receptors and caspase activation}

Death receptors belong to the TNF/NGF receptor family and are characterized by an intracellular death domain that recruits adapter proteins such as TRADD and FADD as well as cysteine proteases such as caspase $8 .^{1} \mathrm{P}$ Schneider (Lausanne, Switzerland) introduced the TNF receptor family which may not only signal apoptosis, but also other biological events including differentiation and even cell survival. Fas ligand activates c-Jun N-terminal kinase only upon extensive aggregation of the receptor and although this is partially dependent on caspase activation, DAXX is not required. DAXX and other death receptor-associated proteins, which have been reported to bind directly or indirectly to Fas such as RIP and RAIDD, were also shown to be dispensable for Fas induced apoptosis. TNF-R1 not only signals cell death, but may also activate the NF- $\kappa$ B pathway resulting in cell survival, two functions which are mutually exclusive. P Schneider also speculated about the structural requirements of the death factors Fas ligand and TRAIL. Both are active as trimers or even higher level aggregates, and their cytotoxicity correlates with their ability to recruit FADD and induce formation of the
DISC. TRAIL contains an unpaired cysteine residue (Cys230) in its receptor-binding domain which is crucial for the biological activity of soluble recombinant TRAIL and cellassociated TRAIL. Mutation of Cys-230 strongly reduced TRAIL's cytotoxic activity and binding to its receptors. In recombinant TRAIL, this residue is engaged either in interchain disulfide bridge formation, resulting in poorly active TRAIL, or in the chelation of one $\mathrm{Zn}^{2+}$ atom per TRAIL trimer in the active form.

$\mathrm{P}$ Vandenabeele (Gent, Belgium) investigated the molecular differences between TNF-induced necrosis and Fas ligand-induced apoptosis. ${ }^{2}$ Fibrosarcoma cells treated with TNF died by necrosis due to excessive formation of reactive oxygen intermediates and membrane permeabilization. In contrast to Fas-induced apoptosis, TNF-induced necrosis did not involve caspase 3 and 7 and cleavage of Bid was hardly detectable. Moreover, the necrotic cells were not phagocytosed by macrophages and exposure of phosphadidylserine was hampered by loss of membrane integrity. Overexpression of $\mathrm{Bcl}-2$ prevented both apoptosis and necrosis, whilst addition of ZVAD in combination with Fas activation prevented apoptosis but not necrosis. Inhibition of caspase 8 also promoted necrosis and it was shown that caspase inhibitors sensitize cells to TNFinduced necrosis by induction of reactive oxygen species. Fas triggered apoptosis via FADD/Mort1 and caspase 8 was blocked by zVAD, which forced cells into necrosis, and it was demonstrated that the death domain of FADD is capable of triggering necrosis.

Erythropoiesis is the formation of proerythroblasts and basophilic, polychromatophilic and orthochromatic erythroblasts to mature erythrocytes from hematopoietic progenitors. $^{3} \mathrm{R}$ de Maria (Roma, Italy) investigated the negative regulation of this process and demonstrated that immature erythroid cells express death receptors including Fas, TNFR1, TRAIL-R1 and TRAIL-R2. Fas ligand and TRAIL produced by mature erythroblasts induced the reversible arrest of erythroblast expansion. The interaction of mature erythroblasts with immature erythroblasts via these death ligands resulted in caspase-mediated degradation of the transcription factor GATA-1, leading to impaired erythroblast development. GATA-1 is especially sensitive to caspase 3,7 and 8 , which are activated upon Fas triggering. Expression of a caspase-resistant GATA-1 mutant completely restored erythroid expansion and 
differentiation in the presence of Fas ligand, indicating the presence of a regulatory feedback between mature and immature erythroblasts through caspase-mediated cleavage of GATA-1. Prevention of GATA-1 cleavage allowed erythroid progenitors to survive and differentiate independently of erythropoietin. Ectopic expression of the transcription factor TAL1/SCL also protected immature erythroblasts from Fas ligand-induced differentiation arrest and uncleavable TAL1/SCL protected GATA-1 from caspase cleavage. For eyrthroid cell expansion to occur in the absence of erythropoietin TAL1/SCL has to interact with its heterodimerization partner E47.

\section{Mitochondria-associated pathways}

Stress-induced apoptosis proceeds through a defined sequence of events that involves mitochondrial cytochrome $c$ release and oligomerization of Apaf-1 followed by the recruitment of caspase $9 .{ }^{4} \mathrm{D}$ Green showed a film visualizing the sequential apoptosis-related events triggered in HeLa cells following treatment with staurosporine. Firstly, cytochrome $c$ was released followed by changes in cellular morphology, including changes in the plasma membrane with the expression of phosphatidylserine residues in the outer leaflet and loss of membrane integrity. If cytochrome $c$ release was induced in the presence of caspase inhibitors, the mitochondrial transmembrane potential initially dissipated but then restored, indicating that these organelles can somehow compensate the loss of cytochrome $c$. If ATP was available, the mitochondrial transmembrane potential was maintained even if large amounts of cytochrome $c$ were released. However, as soon as Apaf-1 consumed ATP to oligomerize and form the apoptosome the transmembrane potential irreversibly dropped.

The structural and functional properties of Bim, a member of a distinct subgroup of pro-apoptotic proteins that only resemble other $\mathrm{Bcl}-2$ family members within the BH3 domain, were introduced by A Strasser (Victoria, Australia). Alternative splicing generates three isoforms, $\mathrm{Bim}_{\mathrm{S}}, \mathrm{Bim}_{\mathrm{L}}$, and $\mathrm{Bim}_{\mathrm{EL}}$, which can neutralize the activity of anti-apoptotic $\mathrm{Bcl}-2$-like proteins through their $\mathrm{BH} 3$ domains. $^{5}$ The Bim isoforms have a hydrophobic $\mathrm{C}$ terminus, localize to intracytoplasmic membranes and induce apoptosis. $\mathrm{Bim}_{\mathrm{L}}$ and $\mathrm{Bim}_{\mathrm{EL}}$ are associated with cytoplasmic structures in lymphocytes, myeloid cells, epithelial cells, neuronal cells, and germ cells. In contrast to apoptosis induced by calcium or cytokine withdrawal, DNA damage- and Fas ligand-induced apoptosis occurred independently of Bim. Most embryos from Bim deficient mice died early and although the remaining mice were liveborn they had 2-5-fold more lymphocytes, macrophages and granulocytes. By 1 year of age Bim-deficient mice accumulated excesses of immunoglobulin-secreting plasma cells and $50 \%$ of the animals succumbed due to autoimmune glomerulonephritis. This finding demonstrates that Bim imposes an important barrier against autoimmunity. Bim deficiency also perturbed thymic $T$ cell development, and numbers of both CD4-CD8 - pro-T cells, mature CD4-CD8+ and CD4+CD8 - T cells were 2-3fold higher than in normal littermates.
During cell death a great number of proteins are released from mitochondria, including DIABLO/Smac, antioxidative enzymes and AIF. ${ }^{6}$ The role of mitochondria as an integrating organelle of pro-apoptotic signals was elucidated by $G$ Kroemer (Villejuif, France). AIF is a caspase-independent death effector which translocates from its localization in the mitochondrial intermembrane space to the cytosol and nucleus. AIF is a flavoprotein equipped with an electron transfer function. It has a 3domain structure and possesses an amino-terminal prosequence, a spacer sequence of approximately 27 amino acids and a carboxyterminal 484 amino acid oxidoreductase domain containing a nuclear localization sequence. Once in the nucleus, AIF caused large scale DNA fragmentation and initial chromatin condensation (stage I). Compared with normal control cells, Apaf-1 or caspase 3-deficient cells did not exhibit oligonucleosomal chromatin digestion and a more advanced pattern of chromatin condensation which is usually seen if caspase activated DNase is involved (stage II). Microinjection of Apaf-1 to these cells lead to caspase-independent cell death characterized by stage I nuclear apoptosis and exposure of phosphatidylserine residues on the cell surface. If cytosolic extracts from apoptotic cells were transferred to normal cells, apoptosis could only be blocked by addition of ICAD and depletion of AIF, whilst in caspase 3-deficient cells depletion of AIF was sufficient to prevent apoptosis. G Kroemer also reported that syncytia arising from the fusion of cells expressing a lymphotropic HIV type 1-encoded envelope glycoprotein with cells expressing the CD4/CXC chemokine receptor 4, undergo spontaneous apoptosis. This process was accompanied by caspase activation, signs of mitochondrial transmembrane permeabilization, and release of cytochrome $c$ and AIF. Release of AIF occurred before that of cytochrome $c$ and before caspases were activated. Microinjection of AIF into syncytia sufficed to trigger caspase-independent cytochrome $c$ release and neutralization of AIF by injection of an antibody prevented all signs of apoptosis.

Although $\mathrm{Bcl}-2$ may have a direct effect on the mitochondrial membrane, it also resides and functions on the ER. ${ }^{7}$ Ch Borner (Freiburg, Germany) used the drug Brefeldin $A$ to trigger the inhibition of secretion and dilation of the ER, which resulted in the caspase 8- and Bidindependent release of cytochrome $c$ from mitochondria. This cytochrome $c$ release was blocked both by wild-type $\mathrm{Bcl}-2$ and a Bcl-2 variant that exclusively localized to the $\mathrm{ER}$, raising the possibility that $\mathrm{Bcl}-2$ may sequester other proteins involved in cytochrome $c$ release. In contrast to $\mathrm{Bcl}-2$, Bcl-xL always localized to mitochondria, which appears to be determined by the $\mathrm{C}$-terminus. Exchange of the $\mathrm{BH} 4$ domains of $\mathrm{Bcl}-2$ and $\mathrm{Bcl}-\mathrm{xL}$ did not alter the localization behavior of the proteins and also fully retained their survival function.

The alpha-helical $\mathrm{BH} 3$ domains of pro-apoptotic Bcl-2 family members mediate their death-promoting activities by binding to the hydrophobic pockets of $\mathrm{Bcl}-2$ and $\mathrm{Bcl}-\mathrm{xL}^{8}{ }^{8}$ This interaction can be inhibited by synthetic compounds offering the possibility to manipulate the life-death rheostat for therapeutic purposes. J Yuan (Boston, MA, USA) used 
a fluorescence polarization assay to measure the binding of fluorescence labeled inhibitory peptides. When bound to a larger molecule the peptide rotated slower and reflected polarizing light, whilst the free peptide, which rotated faster, did not give a measurable signal. Using this technique two compounds $\mathrm{BH} 3 \mathrm{I}-1$ and $\mathrm{BH} 3 \mathrm{I}-2$, differing in their $\mathrm{BH} 3$ binding affinity, were generated. As measured by spectrophotometric analysis the dimerization of $\mathrm{Bcl}-\mathrm{xL}$ and $\mathrm{Bax}$ was efficiently inhibited in the presence of the $\mathrm{BH} 3$ inhibitors. The in vitro $\mathrm{BH} 3$ binding affinity of $\mathrm{BH} 3 \mathrm{I}-1$ and $\mathrm{BH} 3 \mathrm{I}-2$ nicely correlated with their ability to induce apoptosis in vivo.

Inactive caspase 9 is localized within the mitochondrial intermembrane space where it is involved in monitoring mitochondrial cytochrome $c$ release and the subsequent activation of caspase $3 .^{9}$ A Marti (Bern, Switzerland) demonstrated that in mammary epithelial cells a significant fraction of the caspase 9 proform is associated with discrete structures in the nucleus, and that treatment of cells with chemotherapeutic drugs leads to the processing of procaspase 9 and the accumulation of nuclear and cytoplasmic caspase activity. Using caspase 3-deficient MCF-7 cells it was further shown that caspase 8-mediated activation of nuclear caspase 9 strictly requires caspase 3 .

Genotoxic stress triggers the release of mitochondrial cytochrome $c$ and induces the formation of a cytosolic complex (apoptosome) which comprises Apaf-1 and is capable of activating caspase 3 and 9 . S Kharbanda (Boston, MA, USA) demonstrated that heat-shock proteins are involved in the negative regulation of cytochrome $c$ dependent apoptosis by directly interfering in the caspase cascade. $^{10}$ Hsp27 inhibited cytochrome $c$-dependent activation of caspase 3 , but had no effect on cytochrome $c$ release, Apaf-1 or caspase 9 activation. However, Hsp27 associated with caspase 3 and inhibited its activation by caspase 9-mediated proteolysis. Depletion of $\mathrm{Hsp} 27$ in cells also depleted caspase 3 and the treatment of cells with DNA-damaging agents dissociated this complex thereby releasing caspase 3 from its block. S Kharbanda also mentioned that the chaperone Hsp90 forms a cytosolic complex with Apaf-1 thereby inhibiting the formation of the active apoptosome. Depletion of Hsp90 depleted Apaf-1 and inhibited cytochrome $c$-mediated activation of caspase 9. Apoptosis signaling via caspase 9 could be restored when purified Apaf-1 was added to Hsp90 depleted cytosolic extracts. Similar to the situation with Hsp27 treatment of cells with DNA damaging agents dissociated the complex of Hsp90 with its target and released the functional caspase from its block.

\section{Inhibitor of apoptosis proteins (IAPs)}

IAPs belong to a family of proteins containing one or more phylogenetically conserved BIR domains. As reported by D Vaux (Victoria, Australia) two functions, inhibition of apoptosis and regulation of mitosis, can be assigned to members of this family of proteins. The yeast BIR protein Bir1p is involved in chromosome segregation events and interacts with Ndc10p, a component of the yeast kinetochore. ${ }^{11}$ Yeast cells deleted for BIR proteins had a chromosome-loss phenotype which could be completely rescued by overexpression of Ndc10p and fulllength or the C-terminal region of Bir1p. Thus, Bir1p seems to participate in chromosome segregation events either directly or via interaction with kinetochore proteins and these effects are not mediated by the BIR motif. Expression of the human BIR protein survivin is increased at G2-M phase and during mitosis it behaves like the chromosomal passenger protein INCENP, which is chromosomal until metaphase but transfers to the midzone microtubule array and the equatorial cortex during anaphase. Disruption of INCENP or survivin function resulted in lethal defects of cytokinesis, spindle assembly, and sister chromatid separation.

The role of survivin in cell division and apoptosis, and its importance for cancer therapy was addressed by DC Altieri (New Haven, CT, USA). Survivin is the only BIR protein which is expressed in a cell cycle dependent manner. ${ }^{12}$ Its crystal structure shows that it forms a dimer through a symmetric interaction with an intermolecularly bound $\mathrm{Zn}^{2+}$ atom. $\mathrm{Thr}^{34}$ matches the consensus phosphorylation site for the mitotic kinase complex, p34 ${ }^{\text {cdc2 }}$-cyclin B1, and spontaneous caspase-dependent apoptosis was observed after expression of the survivin mutant T34A in HeLa cells. This suggests that regulation of apoptosis by survivin is controlled by $\mathrm{p} 34^{\mathrm{cdc} 2}$ phosphorylation of $\mathrm{Thr}^{34}$ and loss of phosphorylation of survivin resulted in the dislocalization of caspase 9. Moreover, survivin is capable of binding to and inhibiting the activation of caspase-9. Using the Tet-off system it was shown that inhibition of survivin expression inhibited the growth of melanomas in mice and that induction of the p34 binding mutant increased the apoptotic index in tumors. Transgenic mice overexpressing survivin in the skin under the control of the human keratin14 promoter showed markedly reduced levels of apoptosis upon UVB irradiation.

\section{Other regulators and/or pathways}

R Friis (Bern, Switzerland) used a differential display method as a coincidence analysis to identify genes expressed in common in different animal tissues undergoing apoptosis. The gene product DOPA decarboxylase-4 (DDC-4) shows high homology with the cysteine-rich domain ligand-binding domain of the fizzled gene family originally defined as tissue polarity genes in Drosophila. ${ }^{13}$ DDC-4 expression is associated with apoptosis in organs during involution, such as the mammary gland at weaning, the ventral prostate after castration or the luteal cells of the post partum Corpus luteum. As shown in the mammary gland DDC-4 expression was confined to the secretory apoptotic cells and not detectable in surviving stromal cells. Transgenic mice expressing DDC-4 in the mammary gland under the control of the mouse mammary tumor virus long terminal repeat promoter, which stimulates gene expression during late pregnancy and early lactation, revealed apoptosis of up to $70 \%$ of their secretory cells at the day of birth.

Tissue transglutaminase (tTG) is a transamidating enzyme catalyzing the $\mathrm{Ca}^{2+}$-dependent irreversible crosslinking of proteins by dipeptide linkages. This activity is regulated at the post-transcriptional level by GTP, polyamines, and nitric oxide. tTG is upregulated as a 
consequence of apoptosis induction and its overexpression facilitates apoptosis in cells. ${ }^{14}$ Mauro Piacentini (Rome, Italy) addressed the question whether this enzyme regulates the post-translational modification of protein substrates involved in early events during apoptosis. In inducible and constitutively overexpressing cell lines tTG led to mitochondrial hyperpolarization, but did not directly induce the release of cytochrome $c$ or apoptosis. The cells, however, were highly susceptible to apoptosis induced by various apoptotic stimuli. tTG was found being associated with the mitochondrial membrane and mitochondria from tTG-overexpressing cells showed striking differences in their structural and functional integrity. Further analysis identified tTG as BH3-containing protein that induced Bax oligomerization on mitochondria by catalyzing the polymerization of glutathione-s-transferase $\mathrm{P} 1$, thereby releasing its blocking effect on Bax.

Cdk5 is associated with apoptotic cell death in adult and embryonic tissues, and strongly expressed in neurons during development. ${ }^{15}$ The importance of this kinase during cell death and differentiation was addressed by Zahra Zakeri (Flushing, NY, USA). The specificity of Cdk5 expression in dying cells was confirmed in limbs of mutant mice defective in the pattern of interdigital cell death, in limbs with increased interdigital cell death upon treatment with retinoic acid, and by its association with cell death in developing tissues. Mouse embryos treated with cyclophosphamide showed disturbed organogenesis and a distinct pattern of malformations. This dose- and time-dependent effect was accompanied by massive apoptosis and enhanced Cdk5 kinase activity in tissues. The expression of $\mathrm{Cdk} 5$ is regulated by the p35 protein which is also expressed in dying cells. Whilst association of Cdk5 with p35 increased its kinase activity and induced cell death, the complex of Cdk5 and the p35 cleavage product p25 provided a differentiation signal to cells. Cell death involvement of Cdk5 was found to be independent of p53 and detectable also in Apaf-1-deficient cells.

\section{Cell death in non-mammalian cells}

In the nematode $C$. elegans more than 12 genes have been identified that are involved in apoptosis of 131 of the 1090 cells generated during development. ${ }^{16}$ The apoptosis machinery in this species was presented by MO Hengartner (Cold Spring Harbor, NY, USA). Apoptosis in C. elegans involves the activation of CED-3 from the inactive zymogen into the mature protease. This step is mediated by CED-4 and in surviving cells CED-3 and CED-4 are antagonized by CED9, which seems to prevent death by sequestering CED-4 and proCED-3 in an inactive ternary complex. In dying cells CED-9 is, in turn, inactivated by the pro-apoptotic $\mathrm{BH} 3$ domaincontaining protein EGL-1. C. elegans' germ cells die in a similar way as their somatic counterparts. The apotosis machinery, however, is activated by a distinct pathway, as loss of EGL-1 function did not affect germ cell apoptosis and although CED-9 is vital for germ cell survival, a gain-offunction mutation did not affect germ cell death. During germ line development gld genes, which code for cytoplasmic RNAbinding proteins, display differential accumulation and are essential for the inhibition of germ cell proliferation. These proteins act by inducing CED-9 expression whilst at the same time expression of CED-4 and CED-3 is repressed. MO Hengartner also compared the DNA damage response in mammalian cells with that in somatic cells from $C$. elegans. In mammalian cells $\mathrm{p} 53$ plays a central role in the transcriptional activation of repair genes and genes involved in cell cycle arrest, whereas apoptosis occurred mainly in a p53independent manner. Somatic cells of $C$. elegans responded to DNA damage by activation of checkpoint genes (dam-1, mrt-2 rad-5), which induced proliferation arrest and were involved in apoptosis by induction of EGL-1, CED-4 and CED3 expression.

S Kumar (Adelaide, Australia) provided evidence that the main components of the mammalian apoptosis machinery are conserved in insects. ${ }^{17}$ In Drosophila the induction of apoptosis requires three closely linked pro-apoptotic genes, reaper, head involution defective (hid), and grim which can be induced by death signals and inhibited by survival factors. Several homologs of the mammalian cell death machinery were identified in Drosophila: the Apaf-1 homolog DARK, the Bcl-2 homolog Buffy and the FADD homolog dFADD. The Drosophila caspase family comprises DCP-1, which has a substrate specificity similar to that of caspase 3 and can be activated by reaper and grim, STRICA, DREDD, DRONC and DRICE. DRONC contains a caspase recruitment domain and its ectopic expression in cells induced apoptosis which could be inhibited by p35. DRONC functions in the reaper, hid, grim pathway and is regulated by ecdysone. It is processed by DARK and itself activates DIAP1 as well as DRICE and DCP-1 resulting in apoptosis. DRONC ablation inhibited cell death in Drosophila embryos and in DARK mutant flies where DRONC is not processed apoptosis was also blocked. S Kumar also introduced DEBCL, which, like Bax, contains a $\mathrm{BH} 3$ domain required to interact with anti-apoptotic $\mathrm{Bcl}-2$ family members including Buffy. DEBCL was able to bind to the caspases DIAP1 and DARK and its ectopic expression in cells caused apoptosis which was inhibited by p35.

Programmed cell death also occurs in plants during developmental processes such as flower development, embryogenesis, seed germination and response to pathogenic attack. ${ }^{18} \mathrm{M}$ Wang (Leiden, $\mathrm{NL}$ ) reported that during programmed cell death plant cells exhibit the classical features of animal cell apoptosis including cytoplasmic shrinkage, nuclear condensation and DNA fragmentation. Although plant cells have neither pro- nor anti-apoptotic Bcl-2 family members, mammalian Bax was capable of inducing mitochondrial cytochrome $c$ release. Plant cells also do not have true caspases and a cascade of death protease activation has not been identified. Nevertheless, in dying cells PARP was cleaved by caspase 3-like activity in the cytosol.

\section{Clinical implications}

\section{Inflammation}

The accumulation of granulocytes mainly occurs by increased differentiation in the bone marrow and decreased apoptosis at 
inflammatory sites. ${ }^{19,20}$ For both processes overexpression of certain cytokines plays a critical role. However, granulocyte apoptosis is also regulated by death factors and both eosinophilic and neutrophilic granulocytes express all known death receptors. The question raised by $\mathrm{H}-\mathrm{U}$ Simon (Bern, Switzerland) was whether the combination of survival and death factors results in either cell survival or apoptosis. From these studies it turned out that death signaling induced by Fas ligand, TRAIL or TNF is sufficient to abrogate the protective function of GM-CSF and G-CSF on neutrophil apoptosis. Apparently, a tyrosine-based inhibitory motif present in death domains of TNF/NGF superfamily receptors is able to recruit inhibitory phosphatases such as the cytosolic tyrosine phosphatase SHP-1. SHP-1 was shown to dephosphorylate Lyn, a tyrosine kinase which transduces cytokine-mediated anti-apoptotic signals in granulocytes. There are several lines of evidence suggesting that this newly identified Fas ligandand TNF-mediated apoptosis pathway in granulocytes is caspase-independent.

AG Rossi (Edinburgh, UK) demonstrated that neutrophilic and eosinophilic granulocytes have distinct patterns of responsiveness to apoptotic stimuli. Eosinophils undergo rapid apoptosis in response to dexamethasone and increased calcium levels in vitro, whilst the opposite response is observed in neutrophils. ${ }^{21}$ Gliotoxin, a fungal metabolite which inhibits NF- $\kappa \mathrm{B}$ activation, induced caspase-dependent neutrophil apoptosis in a dose-dependent manner and augmented the early pro-apoptotic effect of TNF- $\alpha$, which also blocked the NF- $\kappa$ B survival pathway. Similarly, inhibition of transcription by cycloheximide enhanced TNF- $\alpha$-induced apoptosis, suggesting that NF$\kappa \mathrm{B}$ regulates the production of proteins involved in the survival of neutrophils. At later time points TNF- $\alpha$ stimulation of granulocytes may cause the translocation of $\mathrm{NF}-\kappa \mathrm{B}$ to the nucleus, thereby provoking a survival response which involves the upregulation of anti-apoptotic proteins like Mcl-1 and IAPs.

ALPS was presented as a pathological model for the study of inefficient lymphocyte apoptosis due to dysfunction of the Fas-Fas ligand system (type I ALPS) by F RieuxLaucat (Paris, France). In the majority of ALPS patients the non-malignant accumulation of double negative $T$ lymphocytes and autoimmune phenomena are observed. Moreover, most patients show a crippling mutation in the death domain of Fas but are heterozygous for this defect. ${ }^{22}$ This mutation exerts a dominant-negative effect on the activation of caspase 8 . In patients where the mutation results in a truncated soluble receptor the lymphoproliferative manifestations resolved with age, although the immunological disorders persisted. This indicates that Fas-mediated apoptosis plays a more important role in lymphocyte homeostasis in early childhood than later in life. Mutations in both intra- and extracellular domains of Fas result in loss of apoptosis signaling at the level of the initiator caspases 8 and 10. Cells from these patients, however, were normally sensitive to TRAIL-induced apoptosis. There are several cases of ALPS in which defective Fas-mediated apoptosis is observed without mutations in Fas or Fas ligand (type II ALPS), and the hypothesis was raised that this is due to a complementary defect in caspase 10 .
P Krammer (Heidelberg, Germany) discussed the phenomenon that during an immune response the activation of $T$ cells is accompanied by an increase in cell numbers due to the emergence of apoptosis-resistant cells. $^{23}$ The number of $\mathrm{T}$ cells gradually decreases as cells become more and more sensitive to apoptosis. Interestingly, in this situation IL-2 seems to serve a dual function by stimulating $T$ cell expansion on the one and their death on the other hand. The IL-2 receptor may trigger the PI3 kinase-Akt survival pathway resulting in upregulation of $\mathrm{Bcl}-2$ and induction of proliferation. Its death signal is transduced via JAK1-STAT5, resulting in upregulation of Fas ligand on the cell surface probably mediated by a direct effect of STAT5 on the Fas ligand promoter. Moreover, there seems to be a time-dependent pattern in the expression of anti-apoptotic and pro-apoptotic factors in IL-2 activated $T$ cells. Cells stimulated for only 1 day showed upregulated $\mathrm{Bcl}-\mathrm{xL}$, but no formation of the DISC. DISC, however, was involved in the response to IL-2 6 days after the start of stimulation. $P$ Krammer also addressed the protective role of costimulation during $T$ cell receptor activation. Whilst triggering of the $T$ cell receptor alone induced apoptosis, the simultaneous presence of a costimulatory signal, e.g. provided by CD28, resulted in cell survival. Costimulation of $\mathrm{T}$ cells prevented cell death by upregulating the expression of cFLIP and Bcl-xL and/or inhibiting the expression of Fas ligand. How dysregulated $\mathrm{T}$ cell apoptosis due to an imbalance between $T$ cell receptor and costimulator signaling may contribute to disease was exemplified in patients with sepsis. In contrast to $T$ cells of survivors, which were resistant to Fas-induced apoptosis, $T$ cells of nonsurvivors were incapable of coping with the high antigen load at the site of inflammation and underwent apoptosis.

D Green (San Diego, CA, USA) reported that during rheumatoid arthritis the cartilage and bone structures in joints become destroyed by a massive expansion of synoviocytes. Synoviocytes from these patients show mutations at up to 20 different mutational hotspots in the p53 gene in one joint. Apparently, due to the chronic inflammatory response which occurs over several years and includes the production of mutagenic reactive oxygen species, mutations in the p53 gene arise, resulting in diminished apoptosis and loss of cell cycle arrest. That loss of p53 activity in synoviocytes significantly contributes to the severity of the disease was also shown in p53-deficient mice in which the disease progressed more rapidly. Synoviocytes from these animals not only showed diminished apoptosis and increased growth rate in culture, but also increased expression of the matrix metalloproteinase $13 \mathrm{a}$ which is involved in the digestion of type II collagen.

\section{Cancer}

DNA damage results in the formation of free radicals and upregulation of Fas ligand on the cell surface, a response in which the JNK/SAPK pathway is engaged. KM Debatin (UIm, Germany) demonstrated that treatment of cells with doxor- 
ubicin induced formation of the DISC resulting in apoptosis. Whilst $\mathrm{Bcl}-2$ or $\mathrm{Bcl}-\mathrm{xL}$ could not counteract this process, blockage of the Fas signaling pathway had potential to inhibit apoptosis in these cells. Similarly, treatment of HSV-TK transfected cells with gancyclovir also induced aggregation of Fas and DISC formation in the absence of Fas ligand. ${ }^{24}$ The involvement of the Fas signaling pathway in drug-induced apoptosis, however, was restricted to type I cells. KM Debatin also investigated the mitochondrial pathway of apoptosis upon treatment with chemotherapeutic drugs. In most tumor cells the release of cytochrome $c$ and loss of transmembrane potential occurred in a caspase-dependent manner and was inhibited by $\mathrm{Bcl}-2$ and $\mathrm{Bcl}-\mathrm{xL}$. Caspase 8 seems to play a key role during this process, since neuroectodermal tumor cells, which often lack caspase 8 expression, were found to be resistant to TRAIL- and doxorubicin-induced apoptosis. In these tumor cells betulinic acid (BetA) could prove to be a potent and specific inducer of cell death due to its ability to activate the mitochondrial death pathway. BetA was active against neuroectodermal tumor cells and induced apoptosis independently of p53 and death receptors. Moreover, it had a direct effect on mitochondria, resulting in the release of cytochrome $c$ and AIF. Overexpression of $\mathrm{Bcl}-2$ or $\mathrm{Bcl}-\mathrm{xL}$ blocked loss of the mitochondrial transmembrane potential and cytochrome $c$ release, and conferred resistance to BetA at the level of mitochondrial dysfunction, caspase activation and nuclear fragmentation. Neuroblastoma cells resistant to Fas- or doxorubicin-induced apoptosis remained sensitive to treatment with BetA, suggesting the ability of this compound to bypass some forms of drug resistance.

Modulating the expression of key components of the apoptosis machinery to tip the balance between pro- and anti-apoptotic signals in tumor cells is a rational approach to cancer therapy. The group of $U$ Zangemeister-Wittke (Zürich, Switzerland) generated advanced chemistry antisense oligonucleotides with increased RNA binding affinity, including one which binds to a region of high homology shared by the $\mathrm{Bcl}-2$ and $\mathrm{Bcl}-\mathrm{xL}$ mRNA. ${ }^{25}$ This antisense oligonucleotide was capable of inhibiting the expression of both target genes at the same time, it facilitated apoptosis in tumor cells of diverse histological origin and increased their susceptibility to chemotherapy. As shown in models of established tumor xenografts in mice, following systemic application the bispecific antisense oligonucleotide localized at the tumor site where it downregulated Bcl-2 and $\mathrm{Bcl}-\mathrm{xL}$ expression and inhibited tumor growth.

A further approach to apoptosis-linked cancer therapy is to interfere with the function of proteins engaged in the mitogenic signaling pathway by use of low molecular weight compounds. Protein kinases are a group of molecules which function either upstream or downstream of oncogenes or tumor suppressor genes, and are implicated in the generation and maintenance of the transformed phenotype in many tumors. D Fabbro (Novartis, Basel, Switzerland) showed that the EGF-R tyrosine kinase inhibitor PKI166, which specifically fits into the ATP-binding pocket of EGF receptor family proteins, inhibited growth signaling and induced apoptosis in cells. In vivo, PKI166 sensitized pancreatic carcinoma xenografts to the anti-tumor effect of Gemcitabine and inhibited tumor growth by $85 \%$ in combination with chemotherapy. D Fabbro also reported on the preclinical use of an octamer peptide capable of inhibiting the p53-hdm interaction and inducing p53 tumor suppressor activity in cells.

TRAIL may also be suitable as a selective anticancer agent. ${ }^{26} \mathrm{H}$ Walczak (Heidelberg, Germany) demonstrated that a TRAIL trimer kills $50 \%$ of human tumor cell lines, but does not affect the majority of normal human tissues. TRAIL, however, was toxic to hepatocytes and caused hemorrhagic liver necrosis in mice. Although treatment with TRAIL could cure mice from small tumors, it had little activity against larger tumors. The cascade of events following TRAIL treatment were loss of mitochondrial transmembrane potential, production of reactive oxygen species and DNA fragmentation. Activation of caspase 8 and 3 seemed to occur prior to the release of cytochrome $c$ from mitochondria, implying that mitochondria are not important for TRAIL-induced apoptosis. These data were strengthened by the finding that TRAIL also induced apoptosis in chemoresistant tumors with high $\mathrm{Bcl}-2$ and $\mathrm{Bcl}-\mathrm{xL}$ levels.

\section{Neurodegeneration}

Neurotrophins such as NGF and BDNF mediate survival, differentiation and apoptosis of neurons by binding to two types of cell surface receptors: the Trk tyrosine kinases and the p75 neurotrophin receptor (p75NTR). These receptors, which are often coexpressed on cells, coordinate and modulate the response of neurons to neurotrophins in a way that they cooperate or inhibit each other's function. F Miller (Montreal, Canada) addressed the question how p75NTR signals apoptosis. Trk transmits survival and growth signals via the Ras/PI-3/Akt pathway thereby inhibiting apoptotic proteins. Although $\mathrm{p} 75 \mathrm{NTR}$ can potentiate Trk activity through the activation of NF- $\kappa \mathrm{B}$, in most cases, however, it functions as a ligand-activated pro-apoptotic receptor. The p75NTRmediated activation of the NF- $\kappa$ B pathway is not silenced by coincident TrkA activation. After acute insult p53 was shown to be upregulated and induced apoptosis in sympathetic neurons. In cultured neurons expressing p75 and TrkA, p53 levels were elevated in response to both NGF withdrawal and p75NTR receptor activation. NGF withdrawal also resulted in the elevation of Bax, and adenoviral E1B55K, which destabilizes p53, efficiently rescued neurons from apoptosis. Direct stimulation of the MEKK-JNK pathway had similar effects, p53 and Bax were increased and the subsequent neuronal apoptosis was rescued by E1B55K. Unlike the NF$\kappa \mathrm{B}$ pathway, the JNK-p53 pathway could be completely silenced by TrkA. The p53 family member p73 also plays an important role in neuronal apoptosis. p73 is present in developing neurons primarily as a truncated isoform and its upregulated expression could rescue neurons from apoptosis induced by NGF withdrawal or p53. In p73 deficient mice all isoforms of p73 are deleted and JNK-p53-dependent apoptosis of developing sympathetic neurons was found to be greatly enhanced. This indicates that truncated p73 is an essential anti-apoptotic protein in neurons.

Oligodendrocytes undergo apoptosis during the demyelinating disease 'multiple sclerosis' and are vulnerable to 
hypoxia, ischemia, radiation, TNF- $\alpha$, IFN $-\gamma$ and Fas ligand. M Miura (Osaka, Japan) reported that after hypoxia or treatment with TNF- $\alpha$ cultured oligodendrocytes derived from wild-type mice exhibited significant upregulation of caspase 11 and substantial activation of caspase-3, resulting in apoptotic cell loss. Expression of p35 or elimination of caspase 11 suppressed caspase 3 activation and conferred protection against hypoxic injury and TNF- $\alpha$. This finding was confirmed in p35 transgenic mice which were found to be less susceptible to ischemia-induced injury and also to autoimmune-mediated demyelination. This suggests caspases as promising targets for reducing disease-related brain damage. Genetic studies of neuronal cell death in Drosophila indicated that the Bcl-2/CED 9 family member Drob-1, which like Bax localizes to mitochondria, acts as a positive regulator of cell death in insects. Ectopic expression of Drob-1 in the developing Drosophila eye resulted in a rough-eye phenotype and induced apoptosis accompanied by increased caspase activity. However, Drob-1-induced cell death was not antagonized by p35 indicating that it occurred in both a caspase-dependent and independent manner.

Although TGF- $\beta 1$ has been suggested as an important neuronal survival factor in response to brain injury, ${ }^{27}$ its role in neuronal apoptosis is still unclear. $Y$ Zhu (Marburg, Germany) discussed the effect of TGF- $\beta 1$ on neuronal apoptosis in cultured neurons and in a model of middle cerebral artery occlusion in vivo. Induction of neuronal apoptosis in vitro and in vivo was characterized by upregulation of Bad, which is known to interact with Bcl-2 and $\mathrm{Bcl}-\mathrm{xL}$ to inhibit their function. Treatment of neurons with TGF- $\beta 1$ counteracted staurosporine-induced upregulation of Bad and prevented activation of caspase 3. In vivo, overexpression of TGF- $\beta 1$ in the mouse striatum also inhibited Bad expression in response to ischemia, and reduced the infarct volume and the number of apoptotic neurons.

Apoptotic death of neurons also underlies the spongiform degeneration of the central nervous system. A Aguzzi (Zürich, Switzerland) explained the mechanisms of neuroinvasion during transmissible spongiform encephalopathies, including the bovine spongiform encephalopathy (BSE). The pathogen seems to be the prion protein, which lacks any informational nucleic acid and the transmission of which is thought to occur through conversion of a normal host protein $\mathrm{PrP}^{\mathrm{c}}$ into an abnormal isoform. Although the main pathological changes occur in the brain, the infectious prions are taken up by the gut and first accumulate in lymphoid tissues. In immune-deficient mice the development of clinical disease of scrapie depends on the presence of an intact immune system, and mature B cells and dendritic cells seem to be particularly important. Mice lacking functional follicular dendritic cells do not show prion accumulation in the spleen and only signs of retarded neuroinvasion after inoculation of the scrapie pathogen. However, mice deficient in complement receptor expression were completely resistant to infection with scrapie raising the possibility that prions also have to become opsonized by $B$ cells. Although $B$ cells are likely to be involved in the transport and maturation of prions in a PrP-independent manner, the actual entry into the central nervous system seems to require a PrP-positive tissue and probably proceeds via peripheral nerves.

Apoptosis also plays a role in the pathology of retinitis pigmentosa, a disease characterized by the progressive degeneration of rod and cone photoreceptors. ${ }^{28}$ The molecular events involved in the regulation of light-induced photoreceptor apoptosis were addressed by $\mathrm{M}$ Donovan (Cork, Ireland). Approximately 100 mutations have been encountered within the rhodopsin gene in retinitis pigmentosa. In mice with a targeted disruption of the rhodopsin gene that phenotypically mimics retinitis pigmentosa, cells in the outer nuclear layer of the retina degenerated and completely disappeared upon excessive light exposure. Apoptosis of photoreceptors was caspase 3 -independent and initiated by an early rapid increase in $\mathrm{Ca}^{2+}$ followed by generation of superoxide and loss of mitochondrial transmembrane potential. Inhibition of nitric oxide synthase activity sufficed to prevent all signs of apoptosis, implicating a critical role of nitric oxide in this disease. As one possible mechanism it was suggested that nitric oxide, via guanylate cyclase, raises intracellular free $\mathrm{Ca}^{2+}$ levels which then trigger the mitochondrial pathway of cell death.

\section{Acknowledgements}

We apologize to the many participants whose data were not cited. There were more than eighty posters displayed during the meeting and it was impossible to mention all the contributors due to space limitations. We are grateful to the following companies who helped to finance the conference: Alexis Corporation, Lausen, Switzerland; Becton Dickinson AG, Basel, Switzerland; Catalys AG, Wallisellen, Switzerland; Lucerna-Chem AG, Luzern, Switzerland; R\&D Systems Europe, Abingdon, United Kingdom; AstraZeneca AG, Zug, Switzerland; Beckman Coulter International SA, Nyon, Switzerland; ESSEX Chemie AG, Luzern, Switzerland; LabForce AG, Nunningen, Switzerland; Novartis Pharma AG, Basel, Switzerland; Roche Pharma AG, Reinach, Switzerland; Roche Diagnostics AG, Rotkreuz, Switzerland; Ruwag Handels AG, Zurich, Switzerland, and Merck Sharp \& Dohme-Chibret AG, Glattbrugg, Switzerland. Work in the authors' laboratory is supported by the Swiss National Science Foundation (Grant No. 31-58916.99 to H-U Simon, Grant No. 3140473.94 to $U$ Zangemeister-Wittke), Helmut Horten Foundation ( $\mathrm{H}-\mathrm{U}$ Simon), and Krebsforschung Schweiz (Grant No. 549-0-1997 to U Zangemeister-Wittke).

\section{References}

1. Schneider $P$ and Tschopp $J(2000)$ Apoptosis induced by death receptors. Pharm. Acta Helv. 74: 281-286

2. Fiers W, Beyaert R, Declercq W and Vandenabeele P (1999) More than one way to die: apoptosis, necrosis and reactive oxygen damage. Oncogene 18: 77197730

3. De Maria R, Testa U, Luchetti L, Zeuner A, Stassi G, Pelosi E, Riccioni R, Felli N, Samoggia P and Peschle C (1999) Apoptotic role of Fas/Fas ligand system in the regulation of erythropoiesis. Blood 93: 796-803

4. Waterhouse NJ and Green DR (1999) Mitochondria and apoptosis: HQ or highsecurity prison? J. Clin. Immunol. 19: 378-387

5. Bouillet P, Metcalf D, Huang DC, Tarlinton DM, Kay TW, Kontgen F, Adams JM and Strasser A (1999) Proapoptotic Bcl-2 relative Bim required for certain apoptotic responses, leukocyte homeostasis, and to preclude autoimmunity. Science 286: $1735-1738$ 
6. Brenner $\mathrm{C}$ and Kroemer $\mathrm{G}$ (2000) Apoptosis. Mitochondria-the death signal integrators. Science 289: 1150-1151

7. Hacki J, Egger L, Monney L, Conus S, Rosse T, Fellay I and Borner C (2000) Apoptotic crosstalk between the endoplasmic reticulum and mitochondria controlled by Bcl-2. Oncogene 19: 2286-2295

8. Li H and Yuan J (1999) Deciphering the pathways of life and death. Curr. Opin. Cell Biol. 11: 261-266

9. Blanc C, Deveraux QL, Krajewski S, Janicke RU, Porter AG, Reed JC, Jaggi R and Marti A (2000) Caspase-3 is essential for procaspase-9 processing and cisplatin-induced apoptosis of MCF-7 breast cancer cells. Cancer Res. 60: $4386-4390$

10. Pandey P, Saleh A, Nakazawa A, Kumar S, Srinivasula SM, Kumar V, Weichselbaum R, Nalin C, Alnemri ES, Kufe D and Kharbanda S (2000) Negative regulation of cytochrome c-mediated oligomerization of apaf- 1 and activation of procaspase-9 by heat shock protein 90 . EMBO J. 19: 4310-4322

11. Speliotes EK, Uren A, Vaux D and Horvitz HR (2000) The survivin-like C. elegans BIR-1 protein acts with the Aurora-like kinase AIR-2 to affect chromosomes and the spindle midzone. Mol. Cell 6:211-223

12. Li F, Ackermann EJ, Bennett CF, Rothermel AL, Plescia J, Tognin S, Villa A Marchisio PC and Altieri DC (1999) Pleiotropic cell-division defects and apoptosis induced by interference with survivin function. Nat. Cell Biol. 1:461 466

13. Wolf V, Ke G, Dharmarajan AM, Bielke W, Artuso L, Saurer S and Friis R (1997) DDC-4, an apoptosis-associated gene, is a secreted frizzled relative. FEBS Lett. 417: $385-389$

14. Oliverio S, Amendola A, Rodolfo C, Spinedi A and Piacentini M (1999) Inhibition of "tissue" transglutaminase increases cell survival by preventing apoptosis. J. Biol. Chem. 274: 34123-34128

15. Flaherty DB, Soria JP, Tomasiewicz HG and Wood JG (2000) Phosphorylation of human tau protein by microtubule-associated kinases: GSK3beta and cdk5 are key participants. J. Neurosci. Res. 62: 463-472

16. Liu QA and Hengartner MO (1999) The molecular mechanism of programmed cell death in C. elegans. Ann. N Y Acad. Sci. 887: 92-104

17. Shearwin-Whyatt LM and Kumar S (1999) Caspases in developmental cell death. IUBMB Life 48: 143-150

18. Wang M, Hoekstra S, van Bergen S, Lamers GE, Oppedijk BJ, van der Heijden MW, de Priester W and Schilperoort RA (1999) Apoptosis in developing anthers and the role of $A B A$ in this process during androgenesis in Hordeum vulgare $L$. Plant. Mol. Biol. 39: 489-450
19. Dibbert B, Weber M, Nikolaizik WH, Vogt $P$, Schöni MH, Blaser K and Simon HU (1999) Cytokine-mediated Bax deficiency and consequent delayed neutrophil apoptosis: A general mechanism to accumulate effector cells in inflammation. Proc. Natl. Acad. Sci. USA 96: 13330-13335

20. Simon HU (2000) Eosinophil apoptosis - pathophysiologic and therapeutic implications. Allergy 55: 910-915

21. Ward I, Dransfield I, Chilvers ER, Haslett C and Rossi AG (1999) Pharmacological manipulation of granulocyte apoptosis: potential therapeutic targets. Trends Pharmacol. Sci. 20: 503-509

22. Rieux-Laucat F, Blachere S, Danielan S, De Villartay JP, Oleastro M, Solary E, Bader-Meunier B, Arkwright P, Pondare C, Bernaudin F, Chapel H, Nielsen S, Berrah M, Fischer A and Le Deist F (1999) Lymphoproliferative syndrome with autoimmunity: A possible genetic basis for dominant expression of the clinical manifestations. Blood 94: 2575-2582

23. Holmstrom TH, Schmitz I, Soderstrom TS, Poukkula M, Johnson VL, Chow SC, Krammer PH and Eriksson JE (2000) MAPK/ERK signaling in activated T cells inhibits CD95/Fas-mediated apoptosis downstream of DISC assembly. EMBOJ. 19: $5418-5428$

24. Beltinger C, Fulda S, Kammertoens T, Meyer E, UckertW and Debatin KM (1999) Herpes simplex virus thymidine kinase/ganciclovir-induced apoptosis involves ligand-independent death receptor aggregation and activation of caspases. Proc. Natl. Acad. Sci. USA 96: 8699-8704

25. Zangemeister-Wittke U, Leech SH, Olie RA, Simoes-Wust AP, Gautschi O, Luedke GH, Natt F, Haner R, Martin P, Hall J, Nalin CM and Stahel RA (2000) A novel bispecific antisense oligonucleotide inhibiting both $\mathrm{bcl}-2$ and $\mathrm{bcl}-\mathrm{xL}$ expression efficiently induces apoptosis in tumor cells. Clin. Cancer Res. 6: $2547-2555$

26. Walczak H, Miller RE, Ariail K, Gliniak B, Griffith TS, Kubin M, Chin W, Jones J, Woodward A, Le T, Smith C, Smolak P, Goodwin RG, Rauch CT, Schuh JC and Lynch DH (1999) Tumoricidal activity of tumor necrosis factor-related apoptosisinducing ligand in vivo. Nat. Med. 5: 157-163

27. Zhu Y, Roth-Eichhorn S, Braun N, Culmsee C, Rami A and Krieglstein J (2000) The expression of transforming growth factor-beta1 (TGF-beta1) in hippocampal neurons: a temporary upregulated protein level after transient forebrain ischemia in the rat. Brain Res. 866: 286-298

28. Hobson AH, Donovan M, Humphries MM, Tuohy G, McNally N, Carmody R, Cotter T, Farrar GJ, Kenna PF and Humphries P (2000) Apoptotic photoreceptor death in the rhodopsin knockout mouse in the presence and absence of c-fos. Exp. Eye Res. 71: 247-254 